\title{
A Constant-Power Battery Charger With Inherent Soft Switching and Power Factor Correction
}

\author{
N. K. Poon, Member, IEEE, Bryan M. H. Pong, Senior Member, IEEE, and Chi K. Tse, Senior Member, IEEE
}

\begin{abstract}
A battery charging circuit, which operates as a constant power source, is proposed in this paper. By maintaining a constant output power throughout the charging process, the circuit reduces the size of thermal installation which would normally be required in the cases of constant-voltage or constant-current charging. The proposed circuit takes the form of a half-bridge converter with an additional small inductor and two extra diodes connected in parallel to two dividing capacitors. Constant power delivery is achieved by the discontinuous-voltage-mode operation of the two dividing capacitors, each of which is connected in parallel with a diode. The circuit enjoys low voltage and current stresses, and achieves soft switching with no extra components. When used off-line, the converter maintains a high input power factor and a low level of input current harmonic distortion that meets international regulations. All the above characteristics are determined only by the values of the circuit parameters, the control mechanism being noncritical. A $12 \mathrm{~V} 65 \mathrm{~W}$ prototype was built to demonstrate the merits of this circuit.
\end{abstract}

Index Terms-Battery charger, power factor correction, switching converters, zero-voltage-switching.

\section{INTRODUCTION}

B ATTERY chargers are designed typically around two modes of operation, namely, constant-voltage charging and constant-current charging. The former utilizes a constant voltage source and an equivalent series resistance to control the amount of current that flows into the battery. As soon as the battery voltage is raised to the voltage sources, the converter must limit its current to prevent excessive dissipation. The latter, moreover, keeps the charging current constant until the battery voltage reaches a designated value [1]-[5]. For both modes of charging, mandatory thermal design for the power converter is required. Specifically, in constant-voltage charging, the converter dissipates a large amount of thermal power in the equivalent series resistance, mainly during the initial charging phase. Since this highly dissipative charging phase lasts only for a short duration of the entire charging process, the heavy thermal design, though mandatory, is very sparingly utilized. Moreover, in constant-current charging, the converter delivers very high power when the charging process

Manuscript received December 6, 2000; revised March 23, 2003. Recommended by Associate Editor J. Thottuvelil. This work was supported by the Hong Kong Government under the Innovation and Technology Fund ITS $/ 055 / 01$

N. K. Poon and B. M. H. Pong are with the Department of Electrical and Electronic Engineering, University of Hong Kong, Pokfulam, Hong Kong, China (e-mail: nkpoon@eee.hku.hk; mhp@eee.hku.hk).

C. K. Tse is with the Department of Electronic and Information Engineering, Hong Kong Polytechnic University, Hunghom, Hong Kong, China (e-mail: encktse@polyu.edu.hk).

Digital Object Identifier 10.1109/TPEL.2003.818823 is near completion. Again the high-power charging period only lasts for a short duration of time, the thermal design has a low utilization. In other words, the thermal management for the cases of constant-voltage and constant-current charging must inevitably be over-designed for much of the charging time.

In this paper a circuit topology, which is based on a halfbridge converter topology with an additional small inductor and two diodes connected in parallel with two dividing capacitors, is proposed for battery charging applications. The basic circuit, as studied previously in Pong et al. [6] and Poon et al. [7], [8], has an inherent power limiting characteristic. Moreover, inherent power factor correction (PFC) or harmonic current reduction can be achieved by operation in discontinuous-voltage mode, as demonstrated in Tse [9]. In the proposed circuit, we combine the merits of the above-mentioned topology and the discontinuous-voltage-mode operation, to achieve a very simple design solution that requires minimal thermal installation for battery charging applications. In addition to PFC, soft switching and low voltage stress can be easily achieved with this circuit topology.

\section{OVERVIEW OF POWER LIMITING AND RESISTIVE INPUT CHARACTERISTICS}

Fig. 1 shows the schematic of the constant power converter and some waveforms illustrating the circuit operation. The basic configuration is a half-bridge converter with an additional small inductor $L_{r}$ and two additional diodes connected in parallel to the dividing capacitors $C_{1}$ and $C_{2}$. The duty cycle is fixed and kept at slightly less than $50 \%$ to avoid simultaneous conduction of the two switches. A battery with voltage $V_{T}$ and equivalent series resistance $R_{\mathrm{os}}$ is connected at the output terminals.

The basic operational requirements of the proposed constant power converter are that capacitors $C_{1}$ and $C_{2}$ work in the discontinuous-voltage mode for a given load current, and that the peak voltage across the secondary winding of $T_{1}$ is higher than the battery voltage $V_{T}$ to allow power flow to the battery. Diodes $D_{1}$ and $D_{2}$ limit the voltages of capacitors $C_{1}$ and $C_{2}$ to $0 \mathrm{~V}$ (actually $-0.7 \mathrm{~V}$ ), ensuring that the voltage swing of each capacitor is clamped between the positive supply voltage $V_{i}$ and $0 \mathrm{~V}$.

A main feature of the circuit operation is the discontinuous-voltage-mode operation of the dividing capacitors. When MOSFET $M_{1}$ is on ( $M_{2}$ is off), capacitor $C_{2}$ is charged up and capacitor $C_{1}$ is discharged. The transformer secondary delivers current to the load during this time. As the voltage swing of $C_{1}$ and $C_{2}$ is clamped by diodes $D_{1}$ and $D_{2}$, the voltage at node B will finally reach $V_{i}$ and be clamped at this value. Similarly, 


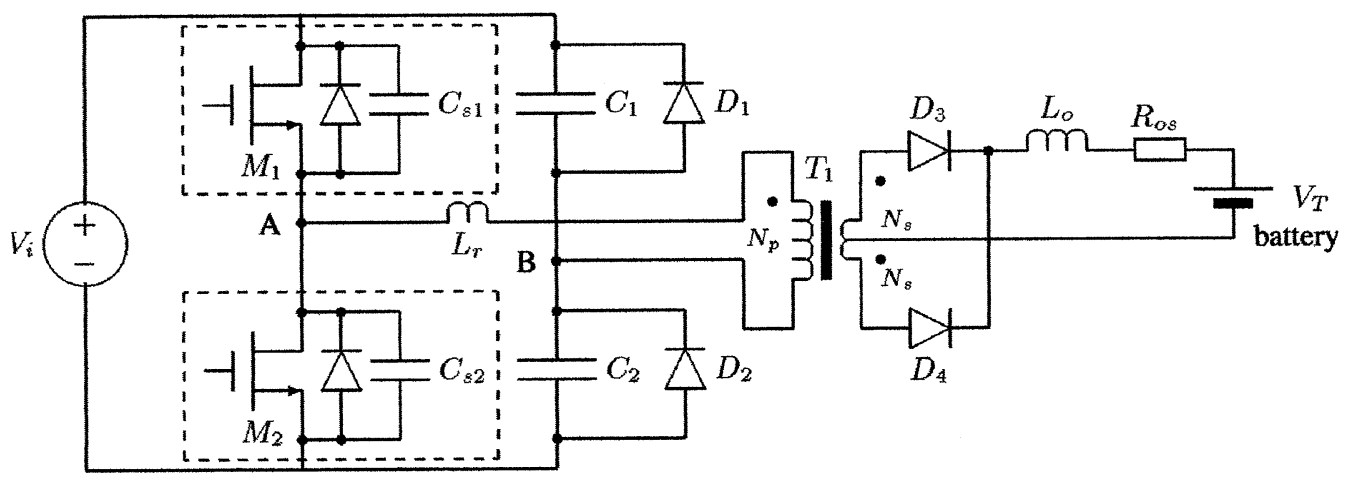

(a)
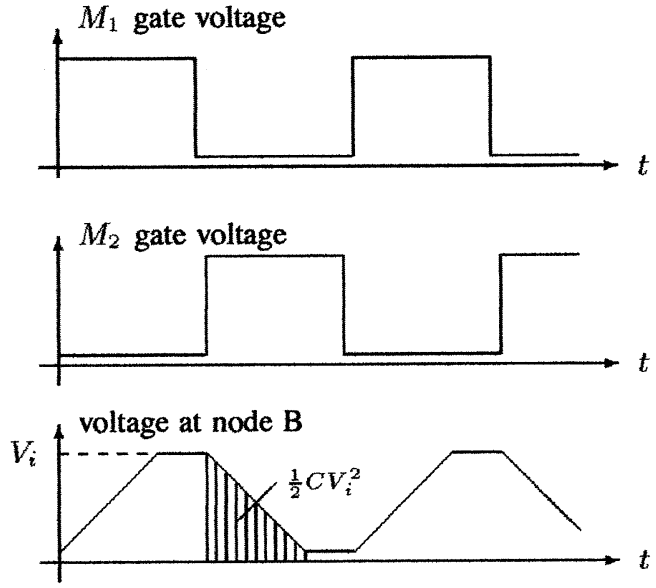

(b)

Fig. 1. Constant power converter for battery charging: (a) schematic and (b) waveforms showing basic operation.

the discharging of $C_{2}$ and charging of $C_{1}$ begin when $M_{2}$ is turned on. In this case, the voltage at node $\mathrm{B}$ will fall to $0 \mathrm{~V}$ and be clamped at this level. Likewise, current is delivered to the output load during the discharging of $C_{2}$ and charging of $C_{1}$. While the detailed operation is left to Section IV, we can immediately appreciate that the output power is limited by the size of capacitors $C_{1}$ and $C_{2}$. Provided that the on-period of $M_{1}$ or $M_{2}$ is sufficiently long, capacitors $C_{1}$ and $C_{2}$ can be charged and discharged fully within a cycle. Thus, the capacitors will transfer their energy entirely to the output side, achieving constant power delivery. Furthermore, the loss associated with discharging and charging the dividing capacitors is partially recovered and delivered to the load due to the presence of an reactive elements $L_{r}$ which is placed in series with the equivalent loading at the primary side of transformer $T_{1}$.

For a preliminary analysis of the circuit operation, we may assume that

a) the primary magnetizing inductance of transformer $T_{1}$ is large enough to make the magnetizing current negligible;

b) the output inductance $L_{O}$ is large enough to maintain a continuous-mode operation of its current.

Suppose that in each switching cycle, the dividing capacitors are completely discharged of the energy stored during the charging interval. Since the stored energy in $L_{r}$ is not delivered directly to the load (rather recovered to the input), the power delivered to the primary side of the transformer, denoted by $P_{T 1}$, is given by

$$
P_{T 1}=\left(C_{1}+C_{2}\right) V_{i}^{2} f_{s}-L_{r} I_{p}^{2} f_{s}
$$

where $I_{p}$ is the output current reflected at the primary winding of transformer $T_{1}$, and $f_{s}$ is the switching frequency. Neglecting the voltage drop across the equivalent series resistance of the battery, the maximum output power $P_{o, \max }$ can be written as

$$
P_{o, \max }=V_{T} I_{p} \frac{N_{p}}{N_{s}} .
$$

Equating $P_{o, \max }$ and $P_{T 1}$ gives

$$
\begin{aligned}
P_{o, \max } & =C_{12} V_{i}^{2} f_{s} \\
& -\frac{\left[\frac{N_{p}}{N_{s}}\left(\sqrt{V_{T}^{2}+4 L_{r} C_{12}\left(f_{s} V_{i} \frac{N_{s}}{N_{p}}\right)^{2}}-V_{T}\right)\right]^{2}}{4 L_{r} f_{s}}
\end{aligned}
$$

where $C_{12}=C_{1}+C_{2}$. If $L_{r} \ll\left(1 / 4 C_{12}\right)\left(V_{T} N_{p} / V_{i} f_{s} N_{s}\right)^{2}$, the maximum output power can be approximated as

$$
P_{o, \max } \approx C_{12} V_{i}^{2} f_{s} \text {. }
$$

The validity of the above approximate formula is well justified because $L_{r}$ is usually small. This is because the purpose of $L_{r}$ is to assist soft switching of the MOSFET switches, and its value 
needs only be large enough to buffer the energy from the equivalent drain-source stray capacitors.

To see the inherent power-factor-correction characteristic, we simply note from (4) that the input impedance of the converter is resistive and is given by $R_{i}=1 / C_{12} f_{s}$. Hence, when used with an $\mathrm{AC}$ mains input, the circuit naturally attains high input power factor.

It should be noted that in order to ensure constant power operation, the output current should be maintained at a level such that the output power falls within the power limit. This puts a limit on the ohmic drop across the connecting wire and the battery internal resistance, i.e.,

$$
R_{\mathrm{os}} \leq \frac{\frac{V_{i} N_{s}}{2 N_{p}}-V_{T}}{2 C_{12} f_{s} V_{i} \frac{N_{p}}{N_{s}}}
$$

where $R_{\mathrm{os}}$ is the equivalent resistance consisting of the battery internal resistance and the connecting wire, and $V_{i}>2 V_{T}\left(N_{p} / N_{s}\right)$. In practice, since $R_{\mathrm{OS}}$ is very small, the criterion stated in (5) should be easily satisfied. Thus, this topology is very suitable for the implementation of a constant power charger.

\section{Averaged Circuit Operation in a Mains CyCle}

In the foregoing section, we have demonstrated the inherent resistive input characteristic that can be exploited for power factor correction. Here, we will consider the operation when the input is connected to an AC mains. Referring to Fig. 2, the operation can be described in terms of five stages, corresponding to five sub-intervals of time during a mains half-cycle. For brevity, we let $v_{i}(t)$ be the input voltage, and $C_{i}$ be the equivalent input capacitor which holds a minimum voltage $V_{r}$ during a finite interval of time near the mains zero cross-over. Note that $C_{i}=C_{12} / 4$ if no extra input filter or bulk capacitor is added.

- Stage 1: $0<t<\tau_{0}$-The input voltage $v_{i}(t)$ is lower than $V_{r}$, resulting in zero input current. Fig. 2(b) shows the equivalent circuit during this stage.

- Stage 2: $\tau_{0}<t<\tau_{1}$-The input voltage is higher than $V_{r}$, but the voltage reflected at the transformer secondary is below $V_{T}$. Fig. 2(c) shows the equivalent circuit. In this stage, the input current starts to energize the series dividing capacitors.

- Stage 3: $\tau_{1}<t<\tau_{2}$-This stage occurs for a short transient duration. Fig. 2(d) shows the corresponding equivalent circuit. The input voltage produces a peak transformer secondary voltage higher than the battery voltage $V_{T}$. Power begins to flow to the output. However, the output voltage is still too low to permit a discontinuousvoltage-mode operation of the dividing capacitors.
- Stage 4: $\tau_{2}<t<\tau_{3}$-This stage accounts for the largest part of the mains half-cycle. The equivalent circuit is shown in Fig. 2(e). In this stage, the converter runs into constant power mode, and the input AC impedance becomes virtually resistive. The transformer primary voltage swings between $0 \mathrm{~V}$ and $v_{i}(t)$, and capacitors $C_{1}$ and $C_{2}$ operate in discontinuous-voltage mode.

- Stage 5: $\tau_{3}<t<\tau_{4}$-In this brief transition stage, as illustrated in Fig. 2(f), the input voltage falls too low to maintain the constant power mode, and the input current drops to zero at $\tau_{4}$. The voltage across $C_{i}$ will stay at $V_{r}$.

Having gone through the various stages in a mains half-cycle, we now derive the formulas for $\tau_{0}, \tau_{1}, \tau_{2}, \tau_{3}, \tau_{4}$, and relevant input and output currents. First of all, $\tau_{0}$ can be found by equating $v_{i}(t)$ and $V_{r}$, i.e.,

$$
\tau_{0}=\frac{\sin ^{-1}\left(V_{r} / \hat{V}_{i}\right)}{\omega_{m}}
$$

where $\hat{V}_{i}$ is the peak input voltage, $\omega_{m}=2 \pi f_{m}$, and $f_{m}$ is the mains frequency. Next, we can find $\tau_{1}$ by noting that during stage 2 , the voltage at the transformer secondary is equal to the battery voltage $V_{T}$, i.e.,

$$
\tau_{1}=\frac{\sin ^{-1}\left(2 V_{T} N_{p} / \hat{V}_{i} N_{s}\right)}{\omega_{m}} .
$$

The input current during stage 2 , denoted by $i_{\left[\tau_{0}-\tau_{1}\right]}(t)$, is given by

$$
i_{\left[\tau_{0}-\tau_{1}\right]}(t)=\omega_{m} C_{i} \hat{V}_{i} \cos \omega_{m} t .
$$

We now consider stage 3 . The output current, denoted by $i_{o\left[\tau_{1}-\tau_{2}\right]}(t)$, is given by (9) shown at the bottom of the page. Since constant power operation starts to come to effect at the end of stage 3 , we can write

$$
i_{o\left[\tau_{1}-\tau_{2}\right]}\left(\tau_{2}\right)=\frac{2 N_{p} C_{12} f_{s} \hat{V}_{i}}{N_{s}} \sin \omega_{m} \tau_{2} .
$$

Furthermore, for $\tau_{1}<t<\tau_{2}$, we have

$$
i_{\left[\tau_{1}-\tau_{2}\right]}(t)=\omega_{m} C_{i} \hat{V}_{i} \cos \omega_{m} t+i_{o\left[\tau_{1}-\tau_{2}\right]}(t) \frac{N_{s}}{2 N_{p}} .
$$

We can therefore find $\tau_{2}$ numerically by putting (9) in (10).

To find $\tau_{3}$, we consider the value of the input voltage at the boundary of constant power mode as given in (5). Since the voltage across the series capacitors $C_{1}$ and $C_{2}$ follow the input voltage, we have

$$
\tau_{3}=\frac{1}{\omega_{m}} \sin ^{-1}\left(\frac{2 V_{T}}{\frac{\hat{V}_{i} N_{s}}{N_{p}}-4 \hat{V}_{i} R_{\mathrm{os}} C_{12} f_{s} \frac{N_{p}}{N_{s}}}\right)
$$

$$
\begin{array}{r}
i_{o\left[\tau_{1}-\tau_{2}\right]}(t)=\frac{\hat{V}_{i} N_{s}}{2 N_{p}}\left(\frac{R_{\mathrm{os}} \sin \omega_{m} t-\omega_{m} L_{o} \cos \omega_{m} t-\left(R_{\mathrm{os}} \sin \omega_{m} \tau_{1}-\omega_{m} L_{o} \cos \omega_{m} \tau_{1}\right) e^{-R_{\mathrm{os}}\left(t-\tau_{1}\right) / L_{o}}}{\omega_{m}^{2} L_{o}^{2}+R_{\mathrm{os}}^{2}}\right) \\
+\frac{V_{T}}{R_{\mathrm{os}}}\left(e^{-R_{\mathrm{os}}\left(t-\tau_{1}\right) / L_{o}}-1\right) .
\end{array}
$$




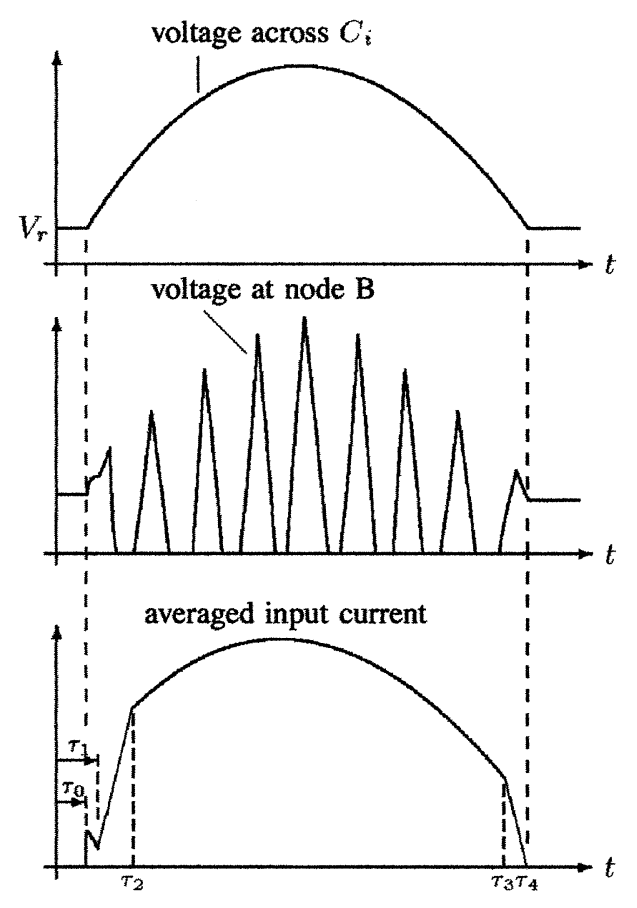

(a)

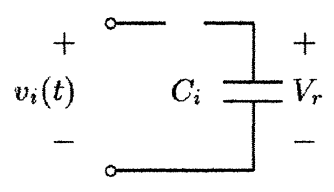

(b)
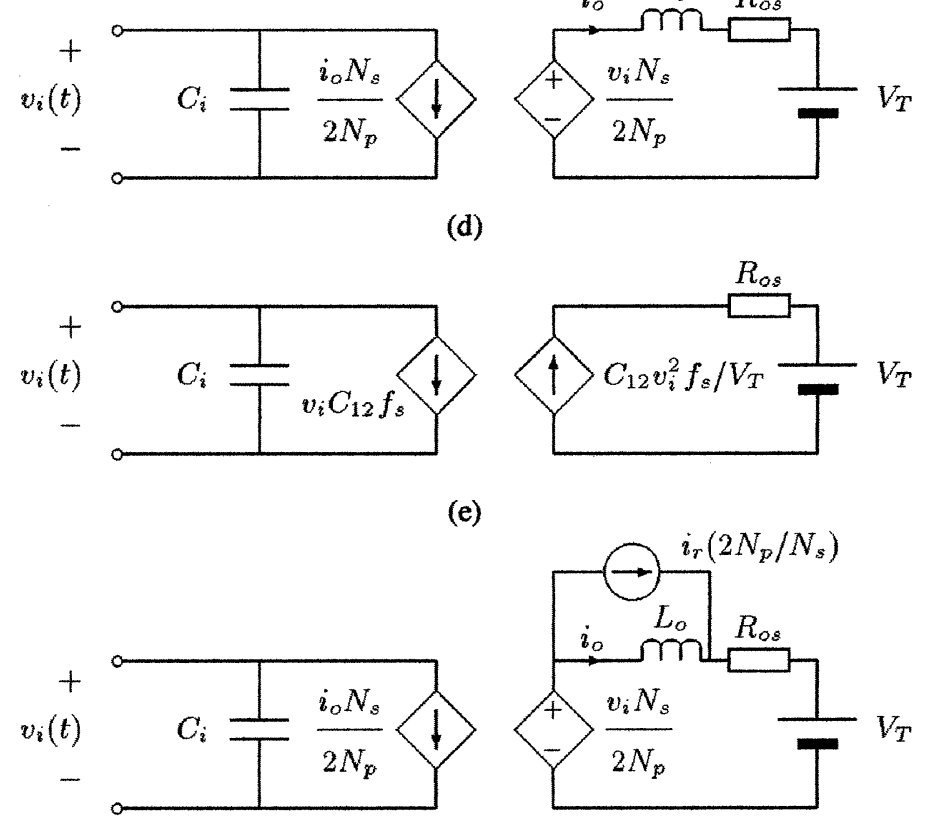

(d)

(e)

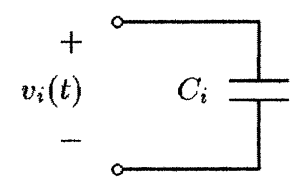

(c)
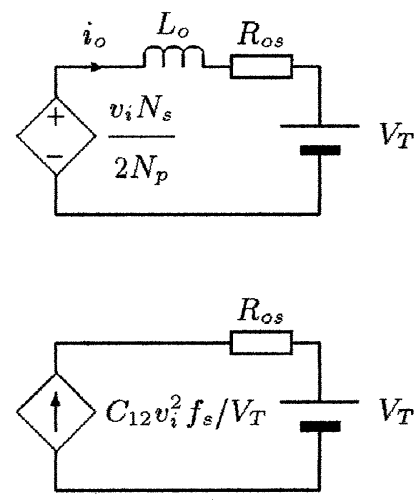

(f)

Fig. 2. (a) Waveforms showing different stages in a mains half-cycle, (b) equivalent averaged circuit for $0<t<\tau_{0}$, (c) equivalent averaged circuit for $\tau_{0}<$ $t<\tau_{1}$, (d) equivalent averaged circuit for $\tau_{1}<t<\tau_{2}$, (e) equivalent averaged circuit for $\tau_{2}<t<\tau_{3}$, and (f) equivalent averaged circuit for $\tau_{3}<t<\tau_{4}$.

for $\left(1 / 4 f_{m}\right)<t<\left(1 / 2 f_{m}\right)$, and the corresponding input current waveform during stage 4 is

$$
i_{\left[\tau_{2}-\tau_{3}\right]}(t)=\omega_{m} C_{i} \hat{V}_{i} \cos \omega_{m} t-C_{12} f_{s} \hat{V}_{i} \sin \omega_{m} t
$$

Also, capacitor $C_{i}$ will hold up the input voltage and prevent the voltage across the capacitor from following the input voltage waveform. From (13), the input current can drop to zero before $\tau_{3}$ is reached, provided that

$$
C_{i}>\left|\frac{C_{12} f_{s} \sin \omega_{m} \tau_{3}}{\omega_{m} \cos \omega_{m} \tau_{3}}\right| .
$$

Suppose the input current falls to zero at $t=\tau_{3}^{\prime}<\tau_{3}$. It is readily found that

$$
\tau_{3}^{\prime}=\frac{-1}{\omega_{m}} \tan ^{-1}\left(\frac{\omega_{m} C_{i}}{C_{12} f_{s}}\right) .
$$

However, in practice, $\omega_{m} \ll 2 \pi f_{s}$ and there is no large input bulk capacitor, (15) therefore has no acceptable solution, meaning that the input current cannot fall to zero before $t=\tau_{3}$.
We can thus assume that at $t=\tau_{3}$ the input current takes a nonzero value of $i_{r}$. The input current waveform during stage 5 is then given by (16) shown at the bottom of the page, and $i_{r}$ can be simply written as

$$
i_{r}=C_{12} f_{s} \hat{V}_{i} \sin \omega_{m} \tau_{3} .
$$

Thus, $\tau_{4}$ can be numerically obtained from

$$
i_{\left[\tau_{3}-\tau_{4}\right]}\left(\tau_{4}\right)=0 \text {. }
$$

Furthermore, the residue voltage $V_{r}$ can be approximated as

$$
V_{r}=\hat{V}_{i} \sin \omega_{m} \tau_{4}
$$

Finally, since $R_{\mathrm{OS}}$ and $L_{r}$ are small, only stage 4 is significant. The averaged (maximum) input power, denoted by $P_{\text {in,max }}$, can be approximated by averaging the input power over the period from $\tau_{2}$ to $\tau_{3}$, giving

$$
P_{\text {in }, \max } \approx 2 C_{12} f_{s} f_{m} \int_{\tau_{2}}^{\tau_{3}} \hat{V}_{i}^{2} \sin ^{2} \omega_{m} t d t .
$$

$$
\begin{array}{r}
i_{\left[\tau_{3}-\tau_{4}\right]}(t)=\frac{\hat{V}_{i} N_{s}^{2}}{4 N_{p}^{2}}\left(\frac{R_{\mathrm{os}} \sin \omega_{m} t-\omega_{m} L_{o} \cos \omega_{m} t-\left(R_{\mathrm{os}} \sin \omega_{m} \tau_{3}-\omega_{m} L_{o} \cos \omega_{m} \tau_{3}\right) e^{-R_{\mathrm{os}}\left(t-\tau_{3}\right) / L_{o}}}{\omega_{m}^{2} L_{o}^{2}+R_{\mathrm{os}}^{2}}\right) \\
+\frac{V_{T} N_{s}}{2 R_{\mathrm{os}} N_{p}}\left(e^{-R_{\mathrm{os}}\left(t-\tau_{3}\right) / L_{o}}-1\right)+i_{r} e^{-R_{\mathrm{os}}\left(t-\tau_{3}\right) / L_{o}}+\omega_{m} C_{i} \hat{V}_{i} \cos \omega_{m} t
\end{array}
$$




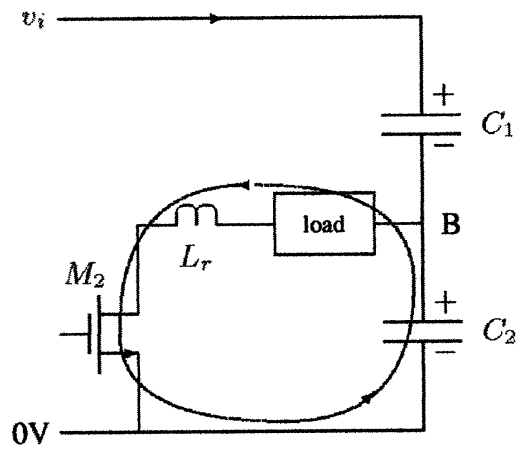

(a)

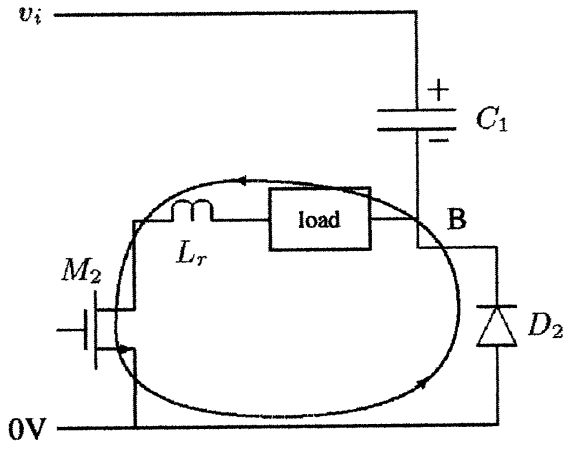

(b)

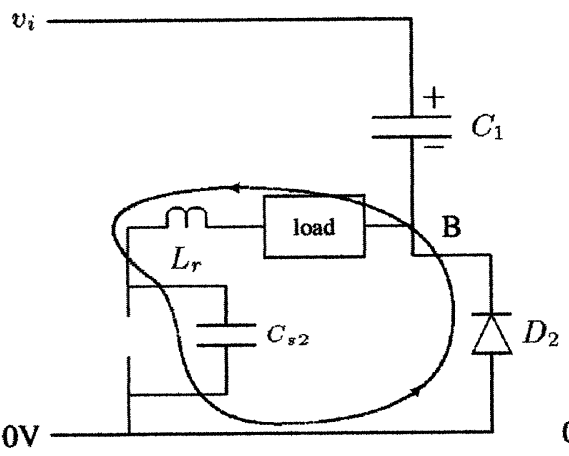

(c)

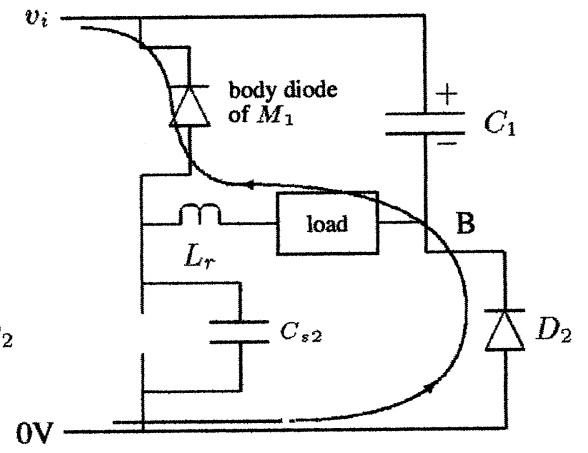

(d)

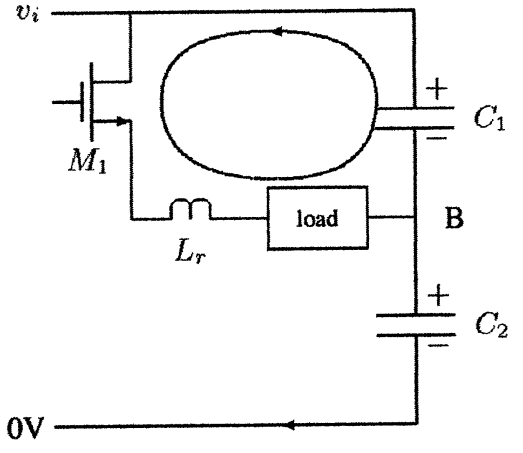

(e)

Fig. 3. Topological sequence in a switching half-cycle for constant power mode $\left(\tau_{2}<t<\tau_{3}\right)$ : (a) $M_{2}$ on and power being delivered to load, (b) current circulating through parallel diode, (c) stray capacitance of $M_{2}$ being charged, (d) zero voltage state created for $M_{1}$, and (e) $M_{1}$ turned on and power being delivered to load.

Expanding gives

$$
\begin{aligned}
P_{\text {in }, \max } \approx & \frac{C_{12} \hat{V}_{i}^{2} f_{s}}{2 \pi}\left(\cos \omega_{m} \tau_{2} \sin \omega_{m} \tau_{2}\right. \\
& \left.\quad-\cos \omega_{m} \tau_{3} \sin \omega_{m} \tau_{3}+\omega_{m} \tau_{3}-\omega_{m} \tau_{2}\right) .
\end{aligned}
$$

The foregoing describes the averaged circuit operation in various stages during a mains half-cycle and provides approximate formulae for the time durations of these stages and the input current waveform which can be used to predict the harmonic contents of the input current. Calculations based on the above formulas and experimental measurements will be compared in a later section.

\section{Circuit Operation in a Switching Cycle}

In this section, we study the detailed circuit operation in a switching cycle, and attempt to highlight the salient characteristics of the proposed converter. Taking advantage of the symmetry of the converter, we need only to consider the half switching cycle [10], [11], and in particular we focus on the duration in which the circuit operates in constant power mode, i.e., $\tau_{2}<t<\tau_{3}$.

Fig. 3 shows the topological sequence in a switching halfcycle. We begin with $M_{2}$ being turned on ( $M_{1}$ off), as illustrated in Fig. 3(a). During this stage, capacitor $C_{2}$ discharges itself and delivers current to the load. The voltage between the two dividing capacitors falls gradually. This stage is the only power delivering stage in the switching half-cycle. Since $L_{r}$ is small and $R_{\mathrm{os}}$ is negligible, the effective duty cycle $D_{\text {eff }}$ can be written as

$$
D_{\text {eff }}(t)=\frac{2 V_{T} N_{p}}{\hat{V}_{i} N_{s} \sin \omega_{m} t}
$$

which is valid as long as the circuit operates in constant power mode, i.e., $\tau_{2}<t<\tau_{3}$ in the mains half-cycle. As capacitor $\mathrm{C}_{2}$ discharges, its voltage falls to $0 \mathrm{~V}$. By action of the small inductor, current forces its way through diode $D_{2}$, as illustrated in Fig. 3(b). The next step is to turn off $M_{2}$. This causes the drain-source capacitance of $M_{2}$ to be charged by the circulating current, as shown in Fig. 3(c) (at the same time discharging that of $M_{1}$ ), and soon the body diode of $M_{1}$ conducts, as shown in Fig. 3(d). This creates a zero-voltage condition for $M_{1}$ to turn on softly [12], [13]. The second half-cycle repeats with the roles of $M_{1}$ and $M_{2}$ interchanged.

It is of interest to know the constraints, if any, on the choice of component values and parameters in order for the circuit to operate as described. Firstly, assuming that the zero-voltage turn-on transient duration corresponding to the stages shown in Fig. 3(c) and (d) is small, the averaged current flowing through diode $D_{2}$, denoted by $i_{D_{2}}(t)$, is

$$
i_{D_{2}}(t)=\left[1-D_{\mathrm{eff}}(t)\right] i_{p}(t)
$$

where

$$
i_{p}(t)=\frac{C_{12} f_{s} N_{s} \hat{V}_{i}^{2} \sin ^{2} \omega_{m} t}{V_{T} N_{p}}
$$




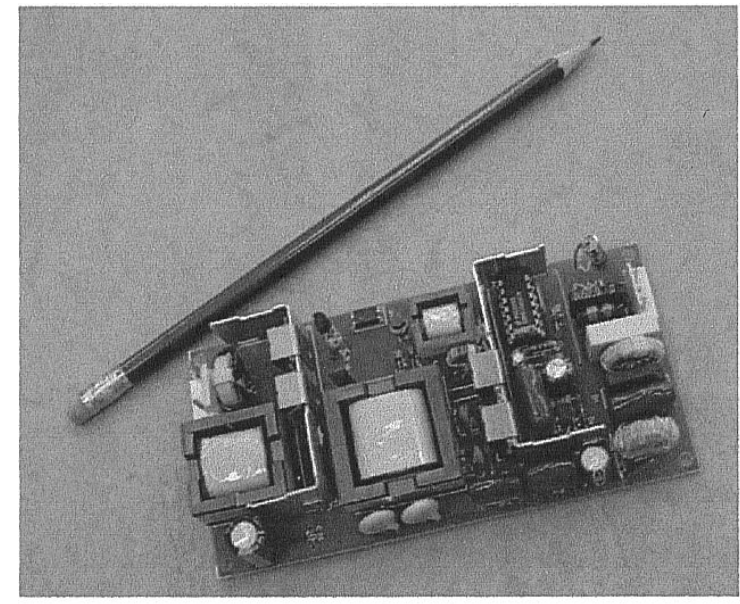

(a)

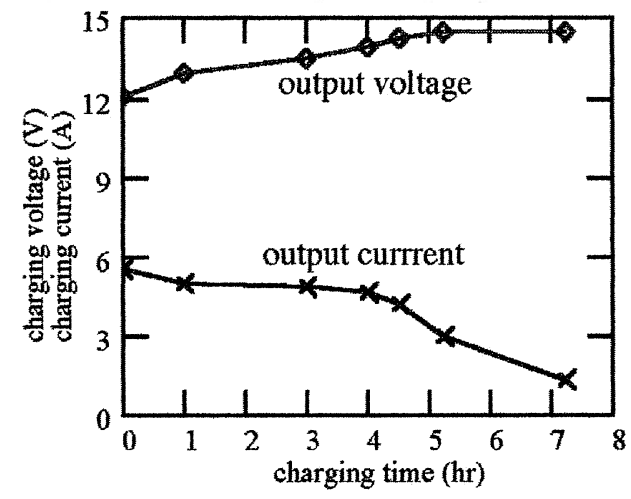

(c)

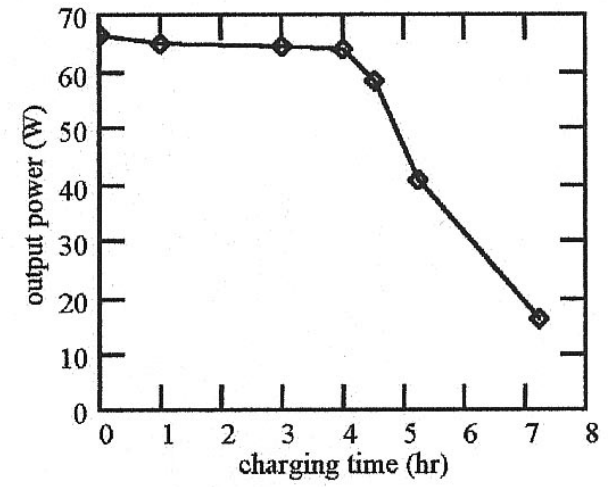

(b)

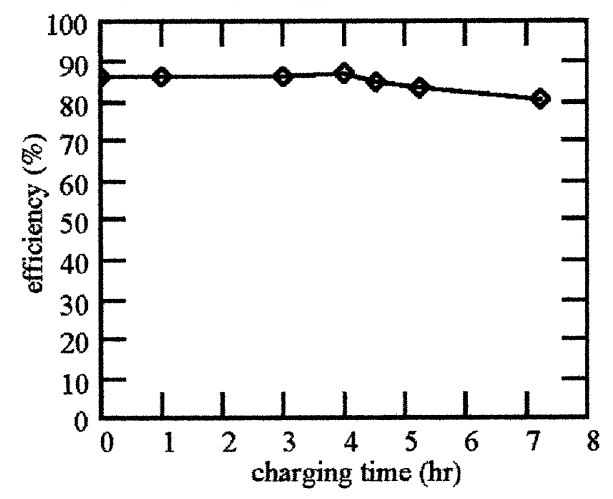

(d)

Fig. 4. (a) Photo of the charger showing minimal thermal requirement, (b) output power, (c) output voltage and current versus charging time, and (d) efficiency versus charging time.

for $\tau_{2}<t<\tau_{3}$ in the mains half-cycle. In fact, $i_{p}(t)$ is the current value at the instant when $M_{2}$ is turned off. Starting with this value, the diode current resonates until a zero-voltage condition is created for $M_{1}$. Let the drain-source capacitance be $C_{s 1}$ and $C_{s 2}$, for $M_{1}$ and $M_{2}$, respectively. Also, define $C_{s}$ by

$$
C_{s}=C_{s 1}+C_{s 2}
$$

which is the equivalent stray capacitance seen at node A (see Fig. 1). Then, the duration of the resonating period, denoted by $\tau_{\text {res }}(t)$, can be written as

$$
\tau_{\text {res }}(t)=\sqrt{L_{r} C_{s}} \sin ^{-1}\left(\frac{V_{T} N_{p}}{C_{12} f_{s} \hat{V}_{i} N_{s} \sin \omega_{m} t} \sqrt{\frac{C_{s}}{L_{r}}}\right)
$$

which is valid for $\tau_{2}<t<\tau_{3}$ in the mains half-cycle. Furthermore, in order for $C_{s 2}$ to be charged up to $v_{i}(t)$ (and $C_{s 1}$ to be discharged to zero), the series inductor should satisfy

$$
L_{r}>C_{s}\left(\frac{V_{T} N_{p}}{C_{12} f_{s} \hat{V}_{i} N_{s} \sin \omega_{m} t}\right)^{2} .
$$

Moreover, we recall from Section II that $L_{r}$ should satisfy

$$
L_{r} \ll \frac{\left(V_{T} N_{p} / \hat{V}_{i} f_{s} N_{s}\right)^{2}}{4 C_{12}}
$$

TABLE I

CALCulated AND Measured Parameter Values

\begin{tabular}{c|rrr}
\hline Parameter & Calculated & Measured & Error \\
\hline$C_{12}$ & $13.58 \mathrm{nF}$ & $13.6 \mathrm{nF}$ & $+0.1 \%$ \\
$P_{\mathrm{in}, \max }$ & $72 \mathrm{~W}$ & $75.7 \mathrm{~W}$ & $+5.1 \%$ \\
$\tau_{0}$ & $2.00 \mathrm{~ms}$ & $1.65 \mathrm{~ms}$ & $-17.5 \%$ \\
$\tau_{1}$ & $2.21 \mathrm{~ms}$ & $1.83 \mathrm{~ms}$ & $-17.2 \%$ \\
$\tau_{2}$ & $2.47 \mathrm{~ms}$ & $2.00 \mathrm{~ms}$ & $-19.0 \%$ \\
$\tau_{3}$ & $7.75 \mathrm{~ms}$ & $8.00 \mathrm{~ms}$ & $+3.2 \%$ \\
$\tau_{4}$ & $7.96 \mathrm{~ms}$ & $8.20 \mathrm{~ms}$ & $+3.0 \%$ \\
$L_{r}$ & $21 \mu \mathrm{H}$ & $20 \mu \mathrm{H}$ & $-4.8 \%$ \\
\hline
\end{tabular}

in order for (4), hence (21), to be valid. Thus, combining (27) and (28), we require that

$$
C_{s} \ll \frac{C_{12}}{4}
$$

which is easily satisfied in most practical cases.

Finally we consider the zero-voltage turn-on condition for the MOSFETs. Basically, $M_{1}$, and $M_{2}$ should be programmed to turn on during a short duration of time in which the drain-source 


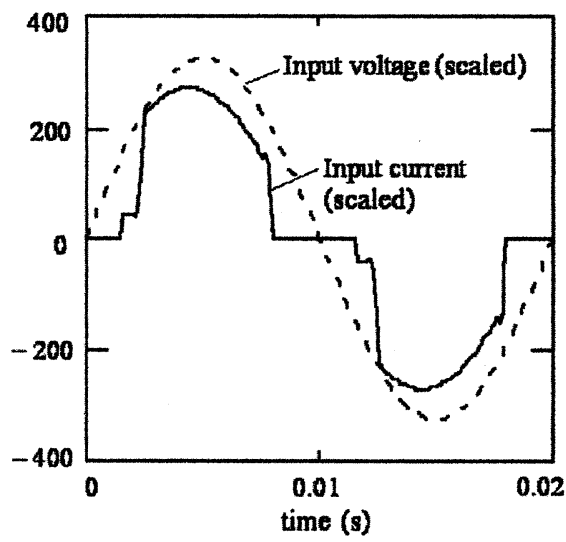

(a)

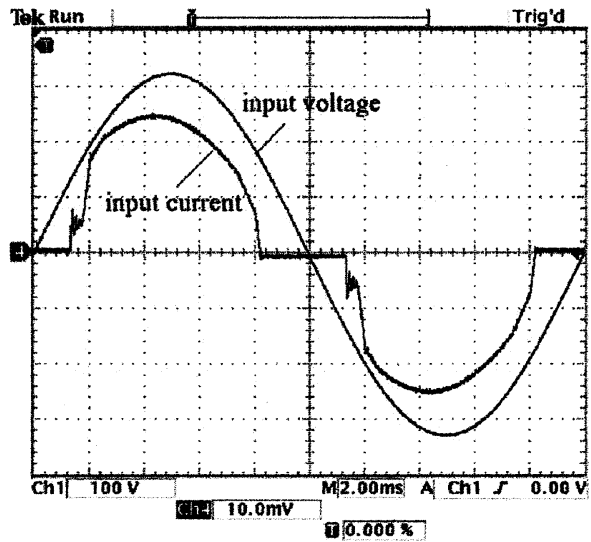

(b)

input current harmonic $(\mathrm{A} / \mathrm{W})$

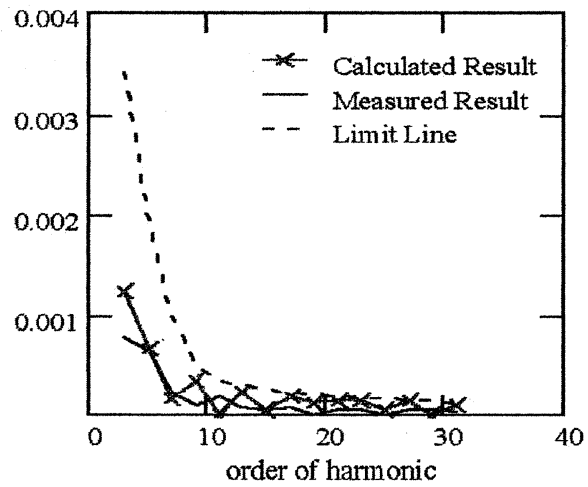

(c)

Fig. 5. (a) Theoretical input current and voltage waveforms, (b) measured input current and voltage waveforms, and (c) calculated and measured input harmonic current contents.

voltage is near zero. Denoted by $\tau_{\mathrm{ZVS}}$, the length of this brief duration can be found as

$$
\tau_{\mathrm{ZVS}}=\left(\frac{C_{12} f_{s} \hat{V}_{i} L_{r} N_{s} \sin \omega_{m} t}{V_{T} N_{p}}\right) \cos \left(\frac{\tau_{\mathrm{res}}(t)}{\sqrt{L_{r} C_{s}}}\right)
$$

which is valid as long as the constant power mode is maintained, i.e., $\tau_{2}<t<\tau_{3}$ in the mains half-cycle.

\section{EXPERIMENTAL VERIFICATION}

A $65 \mathrm{~W}$ constant power charger for a 12 V 35 Ah lead-acid battery was built to verify the proposed design. Assuming a $90 \%$ efficiency, the input power needed is $72 \mathrm{~W}$. The basic circuit parameters are chosen as follows: $f_{s}=120 \mathrm{kHz}$, $N_{s} / N_{p}=3 / 26$, and $C_{\text {in }}=1 \mu \mathrm{F}$ (extra input filtering capacitor). From (21), $C_{12}$ is found as $13.58 \mathrm{nF}$, from which we have $C_{1}=C_{2}=6800 \mathrm{pF}$. The minimum series ZVS inductance, $L_{r}$, is found from (27) as $21 \mu \mathrm{H}$. In the experiment, a $20 \mu \mathrm{H}$ is used. Other crucial components are chosen as follows: diodes $D_{3}$ and $D_{4}$ are MBR20100, MOSFETs $M_{1}$, and $M_{2}$ are STPS6NB50, and transformer core $T_{1}$ is EET30 NC2H. Furthermore, since constant power charging is employed, the charging control is as simple as lowering the power level through the pulse-width modulator once the output voltage has reached the required level.

Table I compares the calculated and measured values of some critical parameters, and Fig. 4 shows a photo of the prototype and some measured results. As shown in Fig. 4(b), the output power remains almost constant for virtually the whole charging period. The power starts to drop at the fifth hour when the battery is fully charged and has reached the clamped voltage of the converter. The converter then further reduces the power by a pulse-width-modulation mechanism. The measured battery voltage $V_{T}$ is $12 \mathrm{~V}$, and the series equivalent resistance $R_{\mathrm{OS}}$ is $0.03 \Omega$. The measured efficiency of the charger reaches $87 \%$, taking into account the loss of an input EMI filter which is needed to suppress the conductive noise to below the class B level.

As shown in Fig. 5(a) and (b), the theoretical and measured waveforms of input voltage and current match quite well. In fact, we have observed that the measured waveform of the input in Fig. 5(b) is even more desirable compared to what has been predicted in Fig. 5(a). The difference is due to the deviation of $\tau_{1}$ and $\tau_{2}$, as a result of the presence of magnetizing inductance of the transformer which causes voltage swing at the center point of the dividing capacitors, giving a higher peak voltage at the secondary of the transformer and an enlarged duration of power 
transfer to the battery. Finally, the input harmonic current contents have been found to meet regulatory requirements, as shown in Fig. 5(c).

\section{CONCLUSION}

A constant power charger with inherent soft switching and power factor correction has been presented. By virtue of the constant charging power, there is no need to install substantial thermal management to address the dissipation problem which may occur only for a short portion of the whole charging process as in the cases of constant voltage and constant current chargers. This advantage will become significant for high-power charging applications. As zero-voltage-switching and power factor correction are achieved inherently with no extra control circuits, the proposed circuit is very suitable for implementation of low-cost high-performance battery chargers.

\section{REFERENCES}

[1] T. Palaniswamy, "Charging techniques for a universal lead-acid battery charger," in Proc. 34th Int. Power Source Conf., 1990, pp. 72-76.

[2] G. E. Mayer, "Overview on batteries and charging methods," in Proc. High Freq. Power Conv. Conf., 1994, pp. 205-214.

[3] C. Gass and E. Tisinger, "Switching current regulator for off-line battery chargers," Proc. IEEE Appl. Power Electron. Conf. Exp., pp. 527-532, 1993.

[4] S. Nonaka and K. Harada, "Characteristics of battery charger using the composite PWM single-phase voltage source converter," in Proc. Jpn Power Electron. Syst. Week Conf. Exh., 1998, pp. 265-270.

[5] T. F. Vescovi and N. C. H. Vun, "A switched-mode 200 A $48 \mathrm{~V}$ rectifier/battery charger for telecommunications applications," in Proc. Int. Telecomm. Energy Conf., 1990, pp. 112-118.

[6] M. H. Pong, W. C. Ho, and N. K. Poon, "Soft switching converter with power limiting feature," Proc. Inst. Elect. Eng., vol. 146, Jan. 1999.

[7] N. K. Poon and M. H. Pong, "Computer aided design of a crossing current resonant converter (XCRC)," in Proc. Ind. Electron. Contr. Inst. Conf., 1994, pp. 135-140.

[8] F. N. K. Poon and B. M. H. Pong, "Capacitor coupled converter," U.S. Patent 5657212 , Aug. 1997.

[9] C. K. Tse, "Zero-order switching networks and their applications to power factor correction," IEEE Trans. Circ. Syst. I, vol. 44, pp. 667-675, Aug. 1997.

[10] A. F. Witulski and R. W. Erickson, "Small signal AC equivalent circuit modeling of the series resonant converter," in Proc. IEEE Electron. Spec. Conf., 1987, pp. 1-12.

[11] S. Korotkov, V. Meleshin, A. Nemchinov, and S. Fraidlin, "Small-signal modeling of soft-switched asymmetrical half-bridge DC/DC converter," Proc. IEEE Appl. Power Electron. Conf. Expo., pp. 707-711, 1995.

[12] J. G. Cho, J. A. Sabate, G. Hua, and F. C. Lee, "Zero-voltage and zerocurrent-switching full bridge PWM converter for high power applications," IEEE Trans. Power Electron., vol. 11, pp. 622-628, July 1996.

[13] W. J. Gu and K. Harada, "A novel self-excited forward DC-DC converter with zero-voltage-switched resonant transitions using a saturable core," IEEE Trans. Power Electron., vol. 10, pp. 131-141, Mar. 1995.

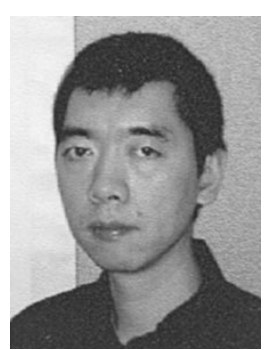

N. K. Poon (M'95) received the B.Eng. (with honors) degree in electronic engineering from the City University of Hong Kong, Kong Kong, in 1995 and the Ph.D. degree from Hong Kong Polytechnic University, Hunghom, Hong Kong, in 2003.

After graduation, he worked with Artesyn Technologies (Asia Pacific), Ltd., for three and a half years before joining the Power Electronics Laboratory, University of Hong Kong. His current interest includes soft switching techniques, EMI modeling, PFC topologies, synchronous rectification, converter modeling, PWM inverters, and fast transient regulators.

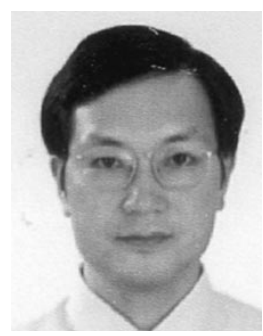

Bryan M. H. Pong (M'84-SM'96) was born in Hong Kong. He received the B.Sc. degree in electronic and electrical engineering from the University of Birmingham, U.K., in 1983 and the Ph.D. degree in power electronics from Cambridge University, U.K., in 1987.

After graduation, he became a Senior Design Engineer and then a Chief Design Engineer at National Semiconductor Hong Kong, where he was involved in electronic product design. Afterwards he joined ASTEC International, Hong Kong, first as a Principal Engineer and then a Division Engineering Manager. He is now an Associate Professor with the University of Hong Kong, where he is in charge of the Power Electronics Laboratory and leads a team to carry out research in switching power supplies. His research interests include synchronous rectification, EMI issues, power factor correction, magnetic component design, and soft switching. He is co-holder of a number of U.S. patents.

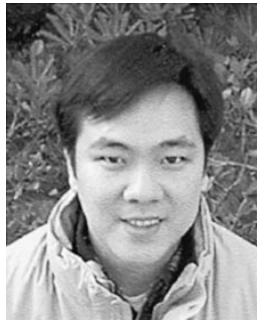

Chi K. Tse (M'90-SM'97) received the B.Eng. (with first class honors) and Ph.D. degrees from the University of Melbourne, Australia, in 1987 and 1991, respectively.

$\mathrm{He}$ is presently a Professor with Hong Kong Polytechnic University, Hong Kong, and his research interests include chaotic dynamics and power electronics. He is the author of Linear Circuit Analysis (London, U.K.: Addison-Wesley 1998) and Complex Behavior of Switching Power Converters (Boca Raton, FL: CRC Press, 2003), co-author of Chaos-Based Digital Communication Systems (Heidelberg, Germany: Springer-Verlag, 2003), and co-holder of a U.S. patent. Since 2002, he has been appointed as Advisory Professor by the Southwest China Normal University, Chongqing, China.

Dr. Tse received the L.R. East Prize by the Institution of Engineers, Australia, in 1987, the IEEE TRANSACTIONS ON POWER Electronics Prize Paper Award, in 2001, the President's Award for Achievement in Research, the Faculty's Best Researcher Award, and a few other teaching awards. He was an Associate Editor for the IEEE TRANSACTIONS ON CIRCUITS AND SYSTEMS PART I-FundAMENTAL THEORY AND APPLICATIONS, from 1999 to 2001, and since 1999 he has been an Associate Editor for the IEEE TRANSACTIONS ON POWER ELECTRONICS. 